\title{
Adult neurogenesis—a reality check
}

\author{
Helena Frielingsdorf • H. Georg Kuhn
}

Received: 1 January 2007 / Accepted: 7 February 2007 / Published online: 8 March 2007

(C) Springer Science + Business Media, LLC 2007

\begin{abstract}
It is established beyond doubt that new neurons are born in discrete areas of the adult brain throughout the lifetime of most mammals. Recent findings have shed new light on the regional limitations, regulation, and possible function of adult neurogenesis. This article aims to look critically at the existence and relevance of adult neurogenesis under physiological conditions, based on recent advances in the field. We also evaluate the therapeutic potential of adult neurogenesis and what is realistic to expect from the future. We conclude that, to date, little is known with certainty about why new neurons are generated in the adult brain. Until there is more causal evidence at hand, assumptions about the potential functions of new neurons remain hypothetical. Provided we learn how to safely regulate proliferation, migration, and proper maturation of new neurons, endogenous neurogenesis could be a promising source of new cells for replacement therapies.
\end{abstract}

Keywords neurogenesis · hippocampus · olfactory bulb · function · therapeutic

H. Frielingsdorf

Neuronal Survival Unit, Department for Experimental Medical Science, Wallenberg Neuroscience Center,

BMC A10, 22184 Lund, Sweden

H. G. Kuhn

Center for Brain Repair and Rehabilitation, Institute for Neuroscience and Neurology, Göteborg University, Box 432, 40530 Göteborg, Sweden

e-mail: georg.kuhn@neuro.gu.se

H. Frielingsdorf $(\square)$

Capio S:t Göran's Hospital, S:t Göransplan 1,

11281 Stockholm, Sweden

e-mail: Helena.Frielingsdorf@med.lu.se

\section{Introduction}

The existence and prospects of adult neurogenesis have stimulated the imaginations of neuroscientists for more than four decades. Initially criticized to be an artifact, it is now considered a fact that adult mammals including rodents and primates produce new neurons from dividing progenitor cells. Adult neurogenesis has been established beyond doubt in two brain regions, the hippocampal dentate gyrus and the subventricular zone (SVZ) of the lateral ventricle wall, through the use of multiple detection methods, such as thymidine labeling, retroviral labeling, and stage specific antibodies. With the development of novel detection techniques, a considerable increase in the amount of studies on various aspects of neurogenesis has emerged during the last 15 years. Numerous experiments have examined whether certain interventions, be it altered gene expression, hormone levels, pharmacological treatments, lesions, or CNS diseases, lead to a change in the numbers of newborn neurons by affecting proliferation, differentiation, and/or survival of the newly generated cells. A number of experiments have focused on how behavioral and environmental variables such as physical exercise, learning paradigms, and enriched environment affect the rate of neurogenesis. Other studies have explored the specific functional characteristics of stem cells, progenitor cells, and new neurons. Several studies have also collected evidence for a wider occurrence of adult neurogenesis in brain regions other than the hippocampus and SVZ and disease situations. This final group of studies has been criticized for their limited methodological repertoire and we will critically evaluate the evidence for neurogenesis outside of the hippocampus and SVZ. We also address the paucity of causal evidence linking new neurons to the potential function(s). Whether new neurons are crucial mediators of information or an irrelevant side effect of 
hippocampal activation remains an open question. What do we really know about the relevance of adult neurogenesis, and can this knowledge be used for the benefit of mankind? Another article in this issue of "Debates in Neuroscience" presents an optimistic perspective on the potentials of adult neurogenesis. Our role in this discussion is to evaluate critically the existence, possible relevance, and therapeutic potential of adult neurogenesis, based on the findings that are at hand to date.

\section{Neurogenesis—where and when?}

At present, only two regions of the adult mammalian brain are generally accepted to harbor endogenous neuronal stem cells; the hippocampal dentate gyrus and the SVZ of the lateral ventricles $[1,2]$. New hippocampal neurons originate from the border between the hilus region and the granule cell layer to form glutamatergic granule cells. Newly formed progenitors from the SVZ migrate to the olfactory bulb to become GABAergic granule cells and GABAergic/ dopaminergic periglomerular interneurons [3]. Beyond these two regions the situation is more controversial. Using in vitro differentiation or transplantation to a neurogenic region, precursor cells with a neurogenic potential have been isolated from the neocortex, septum, striatum, corpus callosum, hypothalamus, substantia nigra, and spinal cord [4-6]. However, in vitro evidence can hardly be used as an argument for actual neurogenesis in the brain, as even the isolated optic nerve or pure astrocyte cultures seem to contain multipotent cells or the ability to be converted to multipotent cells in vitro [6]. We can only infer that cells in these areas have the potential rather than the in vivo function of neural stem cells. The in vivo production of new neurons in the neocortex and amygdala of adult primates [7, 8] is also a matter of dispute [9-11]. Recent studies using postmortem human tissue found no signs of neurogenesis in the adult neocortex $[12,13]$. As the SVZ harbors a high quantity of proliferative neuronal precursors that readily migrate all the way to the olfactory bulb, what is stopping them from also migrating to other areas of the brain? Several studies suggest attractant molecules released from the olfactory bulb [14] and repellent factors in the surrounding tissue [15]. Manipulations such as experimental stroke and growth factor stimulation are suggested to induce migration and neuronal differentiation of SVZ-derived progenitors to anatomically close regions including the striatum, septum, and hypothalamus [16-18]. In this study, stem cell homing factors such as SDF-1 might be involved in directing the migration to the lesion site [19].

Because of its clinical relevance in Parkinson's disease, the adult substantia nigra has been studied in detail with regard to potential production of new neurons. In the adult intact substantia nigra, a few studies found newborn cells with a dopaminergic phenotype in the range of two to ten new dopamine neurons per bilateral substantia nigra per animal [20-22]. On the other hand, several other studies using similar methods found no newborn dopamine neurons in the adult substantia nigra [4, 23, 24].

In summary, most reports on generation of new neurons in areas other than the SVZ/olfactory bulb and hippocampus show a very low rate of neurogenesis. A major critique has been that these studies have exclusively relied on thymidine labeling for the detection of new neurons. Thymidine analogs such as bromo-deoxyuridine (BrdU) are incorporated during DNA synthesis, which occurs during cell division, and also during DNA repair. Therefore, cells undergoing DNA repair while exposed to BrdU may be mistakenly classified by the experimenter as "new cells." Whether the amount of BrdU incorporated during DNA repair is sufficient to be detected by immunohistochemistry is under debate, but it is clearly dependent on the amount of DNA damage in relation to the concentration of BrdU used. At the most commonly used concentration of $50 \mathrm{mg} / \mathrm{kg}$ BrdU, the risk of BrdU uptake into cells undergoing cell death is relatively low in intact tissue, but it increases, for example, after lesions of the nigrostriatal pathway [25].

The best evidence for ongoing neurogenesis is retroviral labeling, as a retrovirus only integrates into the host DNA after nuclear membrane breakdown, which occurs in viable cells only during cell division. The method has the advantage to label all progeny of an infected cell and allows for stable expression of marker proteins such as GFP. It is therefore not only possible to colabel with neuronal markers, but also to prove the functionality of the new neurons using electrophysiological methods [26, 27]. Disadvantages of retroviral labeling include: (1) nonquantitative labeling, (2) invasive intracranial injections, and (3) low frequency of labeling, which makes it a difficult tool to analyze the reportedly rare occurrence of neurogenesis in areas outside the dentate gyrus and SVZ/olfactory bulb.

It is therefore crucial to improve immunohistochemical detection of neurogenesis with BrdU. Prerequisites are proper neuronal markers and the correct use of the confocal microscope to colocalize the immunohistochemical signals, i.e., controlling for bleed-through and optical slice thickness.

What are the best criteria to unmask false colabeling of BrdU with a mature neuronal marker? So far, neurogenesis has been shown to require the cell division of an immature cell, be it a stem cell or a progenitor cell. The maturation into a neuron requires several days in which the cell undergoes frequent morphological and molecular changes, including the expression of stage-specific markers [28]. Hence, one of the key elements in proving neurogenesis in a new region could be the demonstration of this development from an immature cell to a mature neuron. If an experimental paradigm 
includes multiple time points for perfusion after BrdU administration, ranging from a few hours to several weeks, one can determine whether a BrdU signal is detected immediately after BrdU labeling in mature neurons (which would indicate labeling via DNA repair) or whether initially immature marker combinations such as nestin/GFAP or doublecortin colabel with BrdU [29]. The progression through multiple developmental stages is the best proof that neurogenesis is in progress. In conclusion, before a consensus can be reached about the neurogenic potential of different brain areas, we suggest the methodological repertoire for neurogenesis labeling and detection should be verified by at least two independent research groups to meet with well-defined criteria for the definition of an adult-born neuron. Further, we suggest these criteria include at least two out of the following three criteria: 1) detection using retroviral labeling, 2) thymidine analog labeling, and 3) progression through multiple developmental stages using stage-specific markers in combination with proper confocal microscopy.

The analysis of human tissue from postmortem material or surgical resection faces the additional problem that neither BrdU nor retroviral labeling can be routinely performed for ethical reasons, as they have to be administered several weeks or months before tissue collection. Studies have made use of stage-specific markers to prove cell division and immature progenitor cells in similar locations as the nonhuman primate and rodent brain [30-34]. So far, only a single study generated postmortem tissue from patients that received BrdU for in vivo tumor labeling and confirmed the existence of BrdU/NeuN colabeling in the human hippocampus [35]. It is apparent that beyond its existence in the hippocampus, our knowledge on human neurogenesis is limited and mostly extrapolations from primate or rodent studies. However, novel methods such as carbon dating could help answer important questions. This innovative method takes advantage of the dramatic global increase in the levels of $14 \mathrm{C}$ in the atmosphere that took place after testing of nuclear weapons, followed by an exponential decrease after 1963 to roughly determine the DNA synthesis and thereby birth date of cells in tissue samples. The first studies using this technique indicate that there is no turnover of neurons in the adult neocortex, at least in human subjects $[12,13]$.

\section{Function of new neurons in the adult brain under physiological circumstances}

Why are we equipped with the ability to produce new neurons in the dentate gyrus of the adult hippocampus?

The functional relevance of hippocampal neurogenesis can be divided into two issues: (1) at the cellular level, the role of progenitor cells and new neurons within the network and (2) at the behavioral level, the consequences of ongoing neurogenesis for cognitive processes. Numerous publications have characterized the new neurons at the cellular level. Newborn neurons have a lower threshold for eliciting action potentials and long-term potentiation and are more plastic than the existing granule neurons [36, 37]. They are also thought to be added in an activity-dependent manner [38, 39]. Adult-born granule cells follow the same maturation patterns as in embryonic development, including a transient period of being excited, rather than inhibited, by the neurotransmitter GABA the first few weeks after birth $[36,40,41]$. The new neurons in the hippocampus are born in the subgranular zone of the dentate gyrus, from where they migrate a short distance into the granule cell layer, and make dendritic connections with the molecular layer and axonal connections with the CA3 within 2 weeks [42-44]. They become functionally integrated into the existing circuitry, and at 4 to 5 months after mitosis, they appear morphologically indistinguishable from the older granule cells [26] but remain physiologically more responsive to stimulation than the older counterparts [45]. Does this mean that the new neurons can carry out processes the older ones are too "rigid" for? It has been suggested that new neurons come about to avoid "catastrophic interference" in the hippocampus [46], to form temporal links between long-term memories [47], or even to help with the clearance of memories to make room for new ones, i.e., facilitate forgetting [48]. It is also plausible that new neurons strengthen certain forms memory formation while interfering with others, depending on the timing and other factors. However, these theories are at this point hypothetical or based on circumstantial evidence.

\section{Do the numbers matter?}

It is often posited that more new neurons would be beneficial for hippocampal function, but what is the evidence speaking in favor of this assumption? Available immunohistochemical techniques such as Ki-67, PCNA, doublecortin, and thymidine labeling have made it easier to detect and quantify mitotic cells and new neurons. Approximately 9,000 new neurons are generated everyday in the dentate gyrus of a young adult male rat [49]. From this level a continued decline is observed during aging [50], but neurogenesis appears to be still present and inducible at old age [51, 52]. The steepest decline in neurogenesis occurs rather early (during the first year in rats), whereas cognitive impairment that can be associated with age has its onset rather late (after 18 months in rats). Studies attempting to link cognitive performance and numbers of newly born cells in the aged hippocampus have shown very mixed results, one negative and one positive correlation between intact memory function 
and the level neurogenesis in the aged hippocampus and two studies without correlation [53-56].

Regardless of age, about half of the newborn progenitors die within the first few weeks after they are generated $[3,57$, 58]. Those that survive the first critical period are likely to survive for as long as measurements have been made [2, 28].

A functional role for neurogenesis is best defined by experiments that selectively up- or downregulate neurogenesis in the adult brain. As a large number of published studies show, many different manipulations, even seemingly subtle ones such as the amount of handling or the number of BrdU injections, can dramatically reduce or increase the numbers of newborn neurons [59, 60]. Several factors associated with increased cognitive performance also increase neurogenesis, enriched environment [61], voluntary running [62], and restricted diet [63] are some examples. It is crucial to acknowledge that factors that influence the rate of neurogenesis also have a wide array of other effects on the organism, rendering it very difficult to ascertain whether the increase or decrease in neurogenesis contributes to the behavioral effects. Attempts to dissect out the role of neurogenesis in the beneficial effects seen with enriched environment, using ablation techniques, have resulted in diverging findings. One study reports that ablation of hippocampal neurogenesis reduces the beneficial effects of enriched environment on long-term recognition memory [64]. Another recent report indicates that several measures of hippocampus-dependent behavior stimulated by enriched environment occurs independently of hippocampal neurogenesis [65]. However, because of methodological discrepancies, it is difficult to interpret these data. Conversely, a correlation between hippocampus-dependent memory tasks and the numbers of new hippocampal neurons has been sought. Also in this study, the picture has been blurred by diverging results. Engagement in certain hippocampaldependent tasks leads to increased hippocampal neurogenesis [66-69], whereas others lead to no change or even decreased numbers of new neurons [62, 70-72]. A major confounder of these studies is the stress associated with the behavioral tasks, as stress is a powerful regulator of hippocampal neurogenesis [73, 74]. Most hippocampus-dependent memory tasks for rodents involve negative or positive reinforcement, fear, or restraint. However, even if performance of the task is not accompanied by confounders of hippocampal neurogenesis, it is impossible to tell whether an effect on the numbers of new neurons is an effect of the learning per se, or an epiphenomenon elicited by changes in neurotransmitters, blood flow, and other responses to the task.

Different strategies of eliminating adult neurogenesis have been employed to illuminate the importance of intact neurogenesis. Modes of blocking adult neurogenesis in behavioral studies include irradiation, cytostatics, and genetically targeting multipotent neural progenitor cells [75-80]. These studies show no clear-cut effect on cognitive function in general, but interestingly, some measures of specific hippocampus-dependent behaviors are negatively affected. However, the methods for blocking neurogenesis that are currently available are neither brainregion-specific nor cell-type-specific for hippocampal neuronal progenitors. Furthermore, all methods of ablating neurogenesis rely on the elimination of dividing progenitor cells. Even if the ablation was $100 \%$ restricted to the progenitor cells, it should be noted that these progenitor cells are multipotent and thus also give rise to cell types other than neurons, such as astrocytes. Realistically, the effects of ablation may just as well be attributable to the loss of other cell types or factors secreted by them. Development of genetic approaches to conditionally and locally turn down, off, or up neurogenesis in a restricted area could prove to be a more suitable method to study effects on behavior. The different markers used for quantification of new neurons all have their inherent advantages and pitfalls (as discussed above); moreover, they only allow us to study the presence of these markers at one time point, post mortem. Hence, the fate of the new cells cannot be studied over time. There are elegant techniques to study a smaller number of cells using prelabeling of newborn cells in acute hippocampal slices [36, $43,81]$. This makes it possible to study electrophysiological properties and observe development of neuronal processes. However, as the slice is "cut out" of its complete context, it is impossible to study the firing patterns in correlation with the animal's behavior. As discussed above, several studies indicate that interventions that increase hippocampal neurogenesis are paralleled by enhanced cognitive performance. However, as most of these studies acknowledge, the evidence is merely correlational. Taken together, these studies speak in favor of the probability that neurogenesis is a positive modulator of cognition; however, it cannot be ruled out that neurogenesis has nothing to do with the behavioral effects. In conclusion, to date there is no undisputed evidence that links new hippocampal neurons with any changed behavior, mainly because of the fact that there are no techniques available to tease out the exact function of the new neuron. Labeling techniques that allow for in vivo visualization of new cells [82-84] as well as advancements in imaging may provide better tools to study the function of new cells with respect to cognition and behavior.

Are other features more important?

As mentioned above, newly added hippocampal neurons receive and elicit electrical input and seem to participate in the existing circuitry. They also seem to have properties (other than being more recently added to the network) that 
make them different from their older counterparts. Furthermore, the number of new cells born each day make up about $1 \%$ of the total number of granule cells in an adult rat $[49,85]$. However, should all these cells survive, the number of cells in the dentate gyrus would double in just a few months. As most of the newborn cells die within the first 2 weeks after birth, and most of the dying cells in the granule cell layer are newborn $[58,86]$, the major event is new cells competing for survival rather than new cells replacing older ones. Indeed, most recent theories assume that the function of newborn neurons is based on their being new and different from mature cells [47, 87]. So, is the number of new neurons relevant or is it the quality, or "what you do with them" that matters? Are all new neurons the same, or does it matter how they differentiate in terms of dendritic connections, spine formation, axonal projections, phenotype, and receptor expression? The earliest signs and time points of dendritic and axonal projections have been mapped out in a study using real-time confocal imaging [43]. In the same study, it is shown that mice that are allowed to engage in voluntary running develop a richer dendritic arborization faster than nonrunners. In general, newborn neurons are shown to carry receptors for and respond to both GABA and, at a later stage, glutamate [36, $88,89]$. Further, estrogen receptors have been found on only a subset of neurons expressing the early neuronal marker doublecortin [90].

Learning more about the special features of new neurons is necessary to understand whether multiple types of new neurons exist that could contribute to hippocampal processes.

Why neurogenesis in the adult olfactory bulb?

In the intact rodent brain, newly generated cells from the subventricular zone migrate along the rostral migratory stream toward the olfactory bulb. After reaching the bulb, neuroblasts differentiate into amacrine (axonless) interneurons, mainly GABAergic granule cells. Some adult-born cells can be found in the periglomerular layer. They are GABAergic, but about half of them coexpress tyrosine hydroxylase and are also considered dopaminergic [3]. Functionally, it is well established that olfactory deprivation by naris closure decreases neurogenesis in the olfactory bulb, whereas olfactory enrichment increases the generation of new neurons [91, 92]. The effect is mediated through differential survival of newly generated cells in the olfactory bulb [93]. The SVZ proliferation is largely unaffected by olfactory stimulation. From functional analysis in gene knockout studies, which showed altered olfactory neurogenesis, it was concluded that adult neurogenesis is needed for discrimination learning of new odors [92-95]. However, similar to hippocampal studies, whether functional changes are caused by altered olfactory neurogenesis is at this point largely speculative, as a variety of other mechanisms are altered in these knockout models.

At the cellular level the situation is clearer. The new neurons are very much dependent on sensory input and the majority of dying cells in the olfactory bulb appear to be young neurons with dendritic arborization. Olfactory deprivation alters the milieu in the olfactory bulb, for example, by downregulating tenascin- $R$, which in turn leads to reduced recruitment of new neurons [96]. In conclusion, olfactory neurogenesis is clearly olfaction-dependent, but the reverse relation that proper olfactory function needs adult neurogenesis is not fully established. As for the occurrence in higher mammals, it is established that primates also have a rostral migratory stream and olfactory neurogenesis $[10,97,98]$. In humans, the discussion is still open because of conflicting data on new neurons in the olfactory bulb. Although neurogenesis might occur in the olfactory bulb [30], another study reported no structural evidence of a migratory pathway from the ventricle wall [99]. It is therefore too early to postulate the existence of olfactory neurogenesis in humans at this point. Functional speculations on altered olfaction in neurodegenerative diseases as a result of changes in olfactory neurogenesis are therefore also highly speculative.

\section{Adult neurogenesis as a therapeutic tool}

Neural cell transplantation studies have paved the way by showing that lost neurons can be functionally replaced by new ones [100-102]. Endogenous neural stem cells may be a potential source for new neurons. Such an avenue circumvents some of the problems that have plagued tissue transplantation, such as difficulties associated with obtaining donor tissue, surgery, and immunosuppression. In theory, adult neurogenesis promises almost unlimited potential to replace lost cells during neurodegenerative disorders, stroke, and spinal cord injury. After almost two decades of intense research on the regulation of adult neurogenesis, how close are we to taking advantage of endogenous neurogenesis as a therapeutic tool?

One of the most suitable targets for stimulating endogenous neurogenesis is the substantia nigra, as the main neuropathology leading to motor deficits is loss of dopaminergic neurons in the substantia nigra, a single cell type and a single target. Hence, enhancing proliferation and dopaminergic differentiation of local progenitors could be a very attractive means of replacing cells lost in Parkinson's disease. The adult substantia nigra, like many other brain areas, appears to harbor precursor cells that can be coaxed 
into a dopaminergic fate in vitro [4]. The challenge is to develop strategies to induce this potential in vivo to a great enough extent to compensate for the high number of neurons lost in Parkinson's disease. Transcription factors that trigger dopaminergic neurons in the normal immature mesencephalon [103] have recently been identified and could potentially be used to lure immature cells into a dopaminergic phenotype. Then remains the challenging task of ensuring that the newborn dopaminergic neurons make the necessary axonal connection with the striatum to safely deliver the neurotransmitter.

In the adult striatum, neurogenesis has been observed after experimental stroke [16]. Progenitor cells migrate from the SVZ toward the damaged tissue. To a low percentage, differentiation into medium spiny interneurons is observed, a neuronal population which is affected in large numbers by the stroke lesion. This progenitor migration is surprisingly long lasting. Up to 1 year after lesion, the SVZ is still producing a higher number of progenitor cells that enter the striatal parenchyma [19]. However, it is again too early to say whether functional recovery after stroke or trauma is a direct consequence of striatal neurogenesis being present, as no conclusive neurogenesis-ablation studies in combination with stroke lesion have been performed. Moreover, the new neurons only make up about $0.2 \%$ of the numbers of dead neurons after experimental stroke [16], and it has been debated how these few new neurons could significantly impact motor function [104, 105]. However, the extensive progenitor migration to the lesion site could indicate a separate functional role of the progenitor cells, as these cells are likely to produce a variety of growth factors and trophic factors. This might benefit the resident neurons in surviving and in forming new circuitry after a lesion.

Similar to experimental stroke, animal models of epilepsy also increase neurogenesis, particularly in the hippocampus $[106,107]$. It has been suggested that seizure-induced neurogenesis is implicated in the pathological progression of epilepsy [108]. However, another recent study indicates that new neurons generated after seizures show reduced excitability and thus could counteract pathologic epileptic hyperexcitability [109]. Hence, the question of whether generation of new neurons is a detrimental side-effect or a compensatory mechanism to epileptic seizures has to be resolved before adequate therapeutic modulations can be designed.

Similarly, it is unclear whether there is a role for hippocampal neurogenesis in neurodegenerative diseases affecting cognitive function, such as Alzheimer's disease. The characteristic neurodegeneration in Alzheimer's disease is widespread, and cell loss in the hippocampus mainly affects the $\mathrm{CA} 1$ region. Therefore, hippocampal neurogenesis cannot provide a source of neurons primarily to replace lost cell populations. However, as a few newborn hippocampal neurons may change the properties of the entire circuitry, lowering the threshold for excitation, manipulations aimed at enhancing neurogenesis could still be beneficial for cognition. Some studies indicate impaired hippocampal neurogenesis in models for Alzheimer's disease [110-114], and several treatments associated with cognitive improvement have been shown to stimulate hippocampal neurogenesis $[115,116]$. One can therefore speculate, if a cognitive function for neurogenesis exists, a significant loss of neurogenesis during the progression of Alzheimer's disease could contribute to the deterioration of hippocampal function. But again, a causal link between neurogenesis and cognitive function in models of Alzheimer's disease needs to be experimentally established.

Similar to cognition, behavioral effects of antidepressants have been suggested to be dependent on hippocampal neurogenesis [117, 118]. The strongest evidence comes from a study using selective radiation of the hippocampus to demonstrate that the anxiolytic effects of antidepressants are not detectable when hippocampal neurogenesis is eliminated [119]. This study is open to the above-mentioned critique of specificity of the neurogenesis-ablating technique. Nevertheless, it is intriguing that so far most antidepressants tested have neurogenesis-elevating effects when given chronically. But even if behavioral effects of antidepressants require neurogenesis, it is another big step to assume that neurogenesis is involved in the etiology of depression [120]. Here, timing of onset of depression episodes and other variables seem unlikely to be caused by acute dysregulation of hippocampal neurogenesis and further experimental data are needed to provide insight.

\section{Conclusions}

The majority of published work thus far reports that neurogenesis is regulated by an incredible variety of factors. Why is adult neurogenesis so reactive to changes in the internal and external milieu? A possible explanation is that we are observing a large number of developmental processes from proliferation and fate decision, to migration, differentiation, integration, and survival of new neurons. These mechanisms are very sensitive to environmental changes, and this is likely one of the reasons why the largest part of mammalian brain development takes place in the protected environment of the uterus, separated even from the mother by the placenta. Adult neurogenesis is rather exposed and therefore more susceptible to external signals.

Too little is known with certainty about why new neurons are generated in adult brains, how they function, and what their relevance is. The main challenge is to develop techniques to selectively study the function of adult neurogenesis. Before there is more causal evidence at hand, assumptions about the potential functions of new neurons should be made with caution. 
The neurogenic regions could be a source of new cells for cell replacement therapies. Overexpression of factors that drive neuronal differentiation could potentially be used to entice newborn or uncommitted cells located in different brain areas to adopt a neuronal fate. Hence, the therapeutic promise of adult neurogenesis is vast. However, to fully capitalize on the endogenous precursors, signals that trigger cell division, migration, differentiation, and long-term survival have to be controllable from the outside, ideally by pharmacological regulation of these processes.

Acknowledgements We thank the anonymous reviewers for their helpful comments.

\section{References}

1. Altman J (1969) Autoradiographic and histological studies of postnatal neurogenesis. IV. Cell proliferation and migration in the anterior forebrain, with special reference to persisting neurogenesis in the olfactory bulb. J Comp Neurol 137:433-457

2. Altman J, Das GD (1965) Autoradiographic and histological evidence of postnatal hippocampal neurogenesis in rats. J Comp Neurol 124:319-335

3. Winner B, Cooper-Kuhn CM, Aigner R et al (2002) Long-term survival and cell death of newly generated neurons in the adult rat olfactory bulb. Eur J Neurosci 16:1681-1689

4. Lie DC, Dziewczapolski G, Willhoite AR et al (2002) The adult substantia nigra contains progenitor cells with neurogenic potential. J Neurosci 22:6639-6649

5. Markakis EA, Palmer TD, Randolph-Moore L et al (2004) Novel neuronal phenotypes from neural progenitor cells. J Neurosci 24:2886-2897

6. Palmer TD, Markakis EA, Willhoite AR et al (1999) Fibroblast growth factor- 2 activates a latent neurogenic program in neural stem cells from diverse regions of the adult CNS. J Neurosci 19:8487-8497

7. Gould E, Reeves AJ, Graziano MS et al (1999) Neurogenesis in the neocortex of adult primates. Science 286:548-552

8. Bernier PJ, Bedard A, Vinet J et al (2002) Newly generated neurons in the amygdala and adjoining cortex of adult primates. Proc Natl Acad Sci U S A 99:11464-11469

9. Koketsu D, Mikami A, Miyamoto Y et al (2003) Nonrenewal of neurons in the cerebral neocortex of adult macaque monkeys. J Neurosci 23:937-942

10. Kornack DR, Rakic P (2001) Cell proliferation without neurogenesis in adult primate neocortex. Science 294:2127-2130

11. Magavi SS, Leavitt BR, Macklis JD (2000) Induction of neurogenesis in the neocortex of adult mice. Nature 405:951-955

12. Bhardwaj RD, Curtis MA, Spalding KL et al (2006) Neocortical neurogenesis in humans is restricted to development. Proc Natl Acad Sci U S A 103:12564-12568

13. Spalding KL, Bhardwaj RD, Buchholz BA et al (2005) Retrospective birth dating of cells in humans. Cell 122:133-143

14. Liu G, Rao Y (2003) Neuronal migration from the forebrain to the olfactory bulb requires a new attractant persistent in the olfactory bulb. J Neurosci 23:6651-6659

15. Nguyen-Ba-Charvet KT, Picard-Riera N, Tessier-Lavigne $\mathrm{M}$ et al (2004) Multiple roles for slits in the control of cell migration in the rostral migratory stream. J Neurosci 24:1497-1506

16. Arvidsson A, Collin T, Kirik D et al (2002) Neuronal replacement from endogenous precursors in the adult brain after stroke. Nat Med 8:963-970
17. Kokoeva MV, Yin H, Flier JS (2005) Neurogenesis in the hypothalamus of adult mice: potential role in energy balance. Science 310:679-683

18. Pencea V, Bingaman KD, Wiegand SJ et al (2001) Infusion of brain-derived neurotrophic factor into the lateral ventricle of the adult rat leads to new neurons in the parenchyma of the striatum, septum, thalamus, and hypothalamus. J Neurosci 21:6706-6717

19. Thored P, Arvidsson A, Cacci E et al (2006) Persistent production of neurons from adult brain stem cells during recovery after stroke. Stem Cells 24:739-747

20. Shan X, Chi L, Bishop M et al (2006) Enhanced de novo neurogenesis and dopaminergic neurogenesis in the substantia nigra of MPTP-induced Parkinson's disease-like mice. Stem Cells 24:1280-1287

21. Van Kampen JM, Robertson HA (2005) A possible role for dopamine D3 receptor stimulation in the induction of neurogenesis in the adult rat substantia nigra. Neuroscience 136:381-386

22. Zhao M, Momma S, Delfani K et al (2003) Evidence for neurogenesis in the adult mammalian substantia nigra. Proc Natl Acad Sci U S A 100:7925-7930

23. Frielingsdorf H, Schwarz K, Brundin P et al (2004) No evidence for new dopaminergic neurons in the adult mammalian substantia nigra. Proc Natl Acad Sci U S A 101:10177-10182

24. Steiner B, Winter C, Hosman K et al (2005) Enriched environment induces cellular plasticity in the adult substantia nigra and improves motor behavior function in the 6-OHDA rat model of Parkinson's disease. Exp Neurol 199:291-300

25. El-Khodor BF, Oo TF, Kholodilov N et al (2003) Ectopic expression of cell cycle markers in models of induced programmed cell death in dopamine neurons of the rat substantia nigra pars compacta. Exp Neurol 179:17-27

26. van Praag H, Schinder AF, Christie BR et al (2002) Functional neurogenesis in the adult hippocampus. Nature 415:1030-1034

27. Carleton A, Petreanu LT, Lansford R et al (2003) Becoming a new neuron in the adult olfactory bulb. Nat Neurosci 6:507-518

28. Kempermann G, Gast D, Kronenberg G et al (2003) Early determination and long-term persistence of adult-generated new neurons in the hippocampus of mice. Development 130:391-399

29. Cooper-Kuhn CM, Kuhn HG (2002) Is it all DNA repair? Methodological considerations for detecting neurogenesis in the adult brain. Brain Res Dev Brain Res 134:13-21

30. Bedard A, Parent A (2004) Evidence of newly generated neurons in the human olfactory bulb. Brain Res Dev Brain Res 151:159-168

31. Curtis MA, Penney EB, Pearson J et al (2005) The distribution of progenitor cells in the subependymal layer of the lateral ventricle in the normal and Huntington's disease human brain. Neuroscience 132:777-788

32. Bernier PJ, Vinet J, Cossette M et al (2000) Characterization of the subventricular zone of the adult human brain: evidence for the involvement of Bcl-2. Neurosci Res 37:67-78

33. Boekhoorn K, Joels M, Lucassen PJ (2006) Increased proliferation reflects glial and vascular-associated changes, but not neurogenesis in the presenile Alzheimer hippocampus. Neurobiol Dis $24: 1-14$

34. Jin K, Peel AL, Mao XO et al (2004) Increased hippocampal neurogenesis in Alzheimer's disease. Proc Natl Acad Sci U S A 101:343-347

35. Eriksson PS, Perfilieva E, Bjork-Eriksson T et al (1998) Neurogenesis in the adult human hippocampus. Nat Med 4:1313-1317

36. Schmidt-Hieber C, Jonas P, Bischofberger J (2004) Enhanced synaptic plasticity in newly generated granule cells of the adult hippocampus. Nature 429:184-187

37. Snyder JS, Kee N, Wojtowicz JM (2001) Effects of adult neurogenesis on synaptic plasticity in the rat dentate gyrus. $\mathrm{J}$ Neurophysiol 85:2423-2431 
38. Bruel-Jungerman E, Davis S, Rampon C et al (2006) Long-term potentiation enhances neurogenesis in the adult dentate gyrus. $\mathrm{J}$ Neurosci 26:5888-5893

39. Deisseroth K, Singla S, Toda H et al (2004) Excitation-neurogenesis coupling in adult neural stem/progenitor cells. Neuron 42:535-552

40. Ge S, Pradhan DA, Ming GL et al (2007) GABA sets the tempo for activity-dependent adult neurogenesis. Trends Neurosci $30: 1-8$

41. Tozuka Y, Fukuda S, Namba T et al (2005) GABAergic excitation promotes neuronal differentiation in adult hippocampal progenitor cells. Neuron 47:803-815

42. Stanfield BB, Trice JE (1988) Evidence that granule cells generated in the dentate gyrus of adult rats extend axonal projections. Exp Brain Res 72:399-406

43. Zhao C, Teng EM, Summers RG, Jr. et al (2006) Distinct morphological stages of dentate granule neuron maturation in the adult mouse hippocampus. J Neurosci 26:3-11

44. Markakis EA, Gage FH (1999) Adult-generated neurons in the dentate gyrus send axonal projections to field CA3 and are surrounded by synaptic vesicles. J Comp Neurol 406:449-460

45. Ramirez-Amaya V, Marrone DF, Gage FH et al (2006) Integration of new neurons into functional neural networks. J Neurosci 26:12237-12241

46. Wiskott L, Rasch MJ, Kempermann G (2006) A functional hypothesis for adult hippocampal neurogenesis: avoidance of catastrophic interference in the dentate gyrus. Hippocampus 16:329-343

47. Aimone JB, Wiles J, Gage FH (2006) Potential role for adult neurogenesis in the encoding of time in new memories. Nat Neurosci 9:723-727

48. Feng R, Rampon C, Tang YP et al (2001) Deficient neurogenesis in forebrain-specific presenilin-1 knockout mice is associated with reduced clearance of hippocampal memory traces. Neuron 32:911-926

49. Cameron HA, McKay RD (2001) Adult neurogenesis produces a large pool of new granule cells in the dentate gyrus. J Comp Neurol 435:406-417

50. Kuhn HG, Dickinson-Anson H, Gage FH (1996) Neurogenesis in the dentate gyrus of the adult rat: age-related decrease of neuronal progenitor proliferation. J Neurosci 16:2027-2033

51. Kempermann G, Kuhn HG, Gage FH (1998) Experience-induced neurogenesis in the senescent dentate gyrus. J Neurosci 18:3206-3212

52. Kempermann G, Gast D, Gage FH (2002) Neuroplasticity in old age: sustained fivefold induction of hippocampal neurogenesis by long-term environmental enrichment. Ann Neurol 52:135-143

53. Bizon JL, Gallagher M (2003) Production of new cells in the rat dentate gyrus over the lifespan: relation to cognitive decline. Eur J Neurosci 18:215-219

54. Bizon JL, Lee HJ, Gallagher M (2004) Neurogenesis in a rat model of age-related cognitive decline. Aging Cell 3:227-234

55. Merrill DA, Karim R, Darraq M et al (2003) Hippocampal cell genesis does not correlate with spatial learning ability in aged rats. J Comp Neurol 459:201-207

56. Drapeau E, Mayo W, Aurousseau C et al (2003) Spatial memory performances of aged rats in the water maze predict levels of hippocampal neurogenesis. Proc Natl Acad Sci U S A 100:14385-14390

57. Cameron HA, Woolley CS, McEwen BS et al (1993) Differentiation of newly born neurons and glia in the dentate gyrus of the adult rat. Neuroscience 56:337-344

58. Dayer AG, Ford AA, Cleaver KM et al (2003) Short-term and long-term survival of new neurons in the rat dentate gyrus. $J$ Comp Neurol 460:563-572

59. Kempermann G (2005) Regulation Adult Neurogenesis: Stem
Cells and Neuronal Development in the Adult Brain. New York: Oxford University Press

60. Stranahan AM, Khalil D, Gould E (2006) Social isolation delays the positive effects of running on adult neurogenesis. Nat Neurosci 9:526-533

61. Kempermann G, Kuhn HG, Gage FH (1997) More hippocampal neurons in adult mice living in an enriched environment. Nature 386:493-495

62. van Praag H, Kempermann G, Gage FH (1999) Running increases cell proliferation and neurogenesis in the adult mouse dentate gyrus. Nat Neurosci 2:266-270

63. Lee J, Seroogy KB, Mattson MP (2002) Dietary restriction enhances neurotrophin expression and neurogenesis in the hippocampus of adult mice. J Neurochem 80:539-547

64. Bruel-Jungerman E, Laroche S, Rampon C (2005) New neurons in the dentate gyrus are involved in the expression of enhanced long-term memory following environmental enrichment. Eur J Neurosci 21:513-521

65. Meshi D, Drew MR, Saxe M et al (2006) Hippocampal neurogenesis is not required for behavioral effects of environmental enrichment. Nat Neurosci 9:729-73

66. Ambrogini P, Cuppini R, Cuppini C et al (2000) Spatial learning affects immature granule cell survival in adult rat dentate gyrus. Neurosci Lett 286:21-24

67. Dobrossy MD, Drapeau E, Aurousseau C et al (2003) Differential effects of learning on neurogenesis: learning increases or decreases the number of newly born cells depending on their birth date. Mol Psychiatry 8:974-982

68. Gould E, Beylin A, Tanapat P et al (1999) Learning enhances adult neurogenesis in the hippocampal formation. Nat Neurosci 2:260-265

69. Leuner B, Mendolia-Loffredo S, Kozorovitskiy Y et al (2004) Learning enhances the survival of new neurons beyond the time when the hippocampus is required for memory. J Neurosci 24:7477-7481

70. Ehninger D, Kempermann G (2006) Paradoxical effects of learning the Morris water maze on adult hippocampal neurogenesis in mice may be explained by a combination of stress and physical activity. Genes Brain Behav 5:29-39

71. Mohapel P, Mundt-Petersen K, Brundin P et al (2006) Working memory training decreases hippocampal neurogenesis. Neuroscience 142:609-613

72. Van der Borght K, Wallinga AE, Luiten PG et al (2005) Morris water maze learning in two rat strains increases the expression of the polysialylated form of the neural cell adhesion molecule in the dentate gyrus but has no effect on hippocampal neurogenesis. Behav Neurosci 119:926-932

73. Cameron HA, Gould E (1994) Adult neurogenesis is regulated by adrenal steroids in the dentate gyrus. Neuroscience 61:203-209

74. Gould E, McEwen BS, Tanapat P et al (1997) Neurogenesis in the dentate gyrus of the adult tree shrew is regulated by psychosocial stress and NMDA receptor activation. J Neurosci 17:2492-2498

75. Madsen TM, Kristjansen PE, Bolwig TG et al (2003) Arrested neuronal proliferation and impaired hippocampal function following fractionated brain irradiation in the adult rat. Neuroscience 119:635-642

76. Rola R, Raber J, Rizk A et al (2004) Radiation-induced impairment of hippocampal neurogenesis is associated with cognitive deficits in young mice. Exp Neurol 188:316-330

77. Shors TJ, Townsend DA, Zhao M et al (2002) Neurogenesis may relate to some but not all types of hippocampal-dependent learning. Hippocampus 12:578-584

78. Snyder JS, Hong NS, McDonald RJ et al (2005) A role for adult neurogenesis in spatial long-term memory. Neuroscience $130: 843-852$ 
79. Winocur G, Wojtowicz JM, Sekeres M et al (2006) Inhibition of neurogenesis interferes with hippocampus-dependent memory function. Hippocampus 16:296-304

80. Saxe MD, Battaglia F, Wang JW et al (2006) Ablation of hippocampal neurogenesis impairs contextual fear conditioning and synaptic plasticity in the dentate gyrus. Proc Natl Acad Sci U S A 103:17501-17506

81. Wang S, Scott BW, Wojtowicz JM (2000) Heterogenous properties of dentate granule neurons in the adult rat. J Neurobiol 42:248-257

82. Carlen M, Meletis K, Barnabe-Heider F et al (2006) Genetic visualization of neurogenesis. Exp Cell Res 312:2851-2859

83. Couillard-Despres S, Winner B, Karl C et al (2006) Targeted transgene expression in neuronal precursors: watching young neurons in the old brain. Eur J Neurosci 24:1535-1545

84. Overstreet LS, Hentges ST, Bumaschny VF et al (2004) A transgenic marker for newly born granule cells in dentate gyrus. J Neurosci 24:3251-3259

85. West MJ, Gundersen HJ (1990) Unbiased stereological estimation of the number of neurons in the human hippocampus. J Comp Neurol 296:1-22

86. Biebl M, Cooper CM, Winkler J et al (2000) Analysis of neurogenesis and programmed cell death reveals a self-renewing capacity in the adult rat brain. Neurosci Lett 291:17-20

87. Doetsch F, Hen R (2005) Young and excitable: the function of new neurons in the adult mammalian brain. Curr Opin Neurobiol $15: 121-128$

88. Tashiro A, Sandler VM, Toni N et al (2006) NMDA-receptormediated, cell-specific integration of new neurons in adult dentate gyrus. Nature 442:929-933

89. Wadiche LO, Bromberg DA, Bensen AL et al (2005) GABAergic signaling to newborn neurons in dentate gyrus. J Neurophysiol 94:4528-4532

90. Herrick SP, Waters EM, Drake CT et al (2006) Extranuclear estrogen receptor beta immunoreactivity is on doublecortincontaining cells in the adult and neonatal rat dentate gyrus. Brain Res 1121:46-58

91. Corotto FS, Henegar JR, Maruniak JA (1994) Odor deprivation leads to reduced neurogenesis and reduced neuronal survival in the olfactory bulb of the adult mouse. Neuroscience 61:739-744

92. Rochefort C, Gheusi G, Vincent JD et al (2002) Enriched odor exposure increases the number of newborn neurons in the adult olfactory bulb and improves odor memory. J Neurosci 22: $2679-2689$

93. Rochefort C, Lledo PM (2005) Short-term survival of newborn neurons in the adult olfactory bulb after exposure to a complex odor environment. Eur J Neurosci 22:2863-2870

94. Gheusi G, Cremer H, McLean H et al (2000) Importance of newly generated neurons in the adult olfactory bulb for odor discrimination. Proc Natl Acad Sci U S A 97:1823-1828

95. Enwere E, Shingo T, Gregg C et al (2004) Aging results in reduced epidermal growth factor receptor signaling, diminished olfactory neurogenesis, and deficits in fine olfactory discrimination. J Neurosci 24:8354-8365

96. Saghatelyan A, de Chevigny A, Schachner M et al (2004) Tenascin- $\mathrm{R}$ mediates activity-dependent recruitment of neuroblasts in the adult mouse forebrain. Nat Neurosci 7:347-356

97. Koketsu D, Furuichi Y, Maeda M et al (2006) Increased number of new neurons in the olfactory bulb and hippocampus of adult nonhuman primates after focal ischemia. Exp Neurol 199:92-102

98. Pencea V, Bingaman KD, Freedman LJ et al (2001) Neurogenesis in the subventricular zone and rostral migratory stream of the neonatal and adult primate forebrain. Exp Neurol 172:1-16

99. Sanai N, Tramontin AD, Quinones-Hinojosa A et al (2004) Unique astrocyte ribbon in adult human brain contains neural stem cells but lacks chain migration. Nature 427:740-744
100. Bjorklund A, Stenevi U (1979) Reconstruction of the nigrostriatal dopamine pathway by intracerebral nigral transplants. Brain Res 177:555-560

101. Lindvall O, Hagell P (2000) Clinical observations after neural transplantation in Parkinson's disease. Prog Brain Res 127: 299-320

102. Piccini P, Brooks DJ, Bjorklund A et al (1999) Dopamine release from nigral transplants visualized in vivo in a Parkinson's patient. Nat Neurosci 2:1137-1140

103. Andersson E, Tryggvason U, Deng Q et al (2006) Identification of intrinsic determinants of midbrain dopamine neurons. Cell 124:393-405

104. Komitova M, Mattsson B, Johansson BB et al (2005) Enriched environment increases neural stem/progenitor cell proliferation and neurogenesis in the subventricular zone of stroke-lesioned adult rats. Stroke 36:1278-1282

105. Nygren J, Wieloch T, Pesic J et al (2006) Enriched environment attenuates cell genesis in subventricular zone after focal ischemia in mice and decreases migration of newborn cells to the striatum. Stroke 37:2824-2829

106. Bengzon J, Kokaia Z, Elmér E et al (1997) Apoptosis and proliferation of dentate gyrus neurons after single and intermittent limbic seizures. Proc Natl Acad Sci U S A 94:10432-10437

107. Parent JM, Yu TW, Leibowitz RT et al (1997) Dentate granule cell neurogenesis is increased by seizures and contributes to aberrant network reorganization in the adult rat hippocampus. J Neurosci 17:3727-3738

108. Parent JM, Elliott RC, Pleasure SJ et al (2006) Aberrant seizureinduced neurogenesis in experimental temporal lobe epilepsy. Ann Neurol 59:81-91

109. Jakubs K, Nanobashvili A, Bonde S et al (2006) Environment matters: synaptic properties of neurons born in the epileptic adult brain develop to reduce excitability. Neuron 52:1047-1059

110. Chevallier NL, Soriano S, Kang DE et al (2005) Perturbed neurogenesis in the adult hippocampus associated with presenilin-1 A246E mutation. Am J Pathol 167:151-159

111. Dong H, Goico B, Martin M et al (2004) Modulation of hippocampal cell proliferation, memory, and amyloid plaque deposition in APPsw (Tg2576) mutant mice by isolation stress. Neuroscience 127:601-609

112. Donovan MH, Yazdani U, Norris RD et al (2006) Decreased adult hippocampal neurogenesis in the PDAPP mouse model of Alzheimer's disease. J Comp Neurol 495:70-83

113. Haughey NJ, Nath A, Chan SL et al (2002) Disruption of neurogenesis by amyloid beta-peptide, and perturbed neural progenitor cell homeostasis, in models of Alzheimer's disease. J Neurochem 83:1509-1524

114. Jin K, Galvan V, Xie L et al (2004) Enhanced neurogenesis in Alzheimer's disease transgenic (PDGF-APPSw,Ind) mice. Proc Natl Acad Sci U S A 101:13363-13367

115. Jin K, Xie L, Mao XO et al (2006) Alzheimer's disease drugs promote neurogenesis. Brain Res 1085(1):183-188

116. Mohapel P, Leanza G, Kokaia M et al (2005) Forebrain acetylcholine regulates adult hippocampal neurogenesis and learning. Neurobiol Aging 26:939-946

117. Jacobs BL, van Praag H, Gage FH (2000) Adult brain neurogenesis and psychiatry: a novel theory of depression. Mol Psychiatry 5:262-269

118. Duman RS, Nakagawa S, Malberg J (2001) Regulation of adult neurogenesis by antidepressant treatment. Neuropsychopharmacology 25:836-844

119. Santarelli L, Saxe M, Gross C et al (2003) Requirement of hippocampal neurogenesis for the behavioral effects of antidepressants. Science 301:805-809

120. Henn FA, Vollmayr B (2004) Neurogenesis and depression: etiology or epiphenomenon? Biol Psychiatry 56:146-150 